\title{
DIOS: the Diffuse Intergalactic Oxygen Surveyor
}

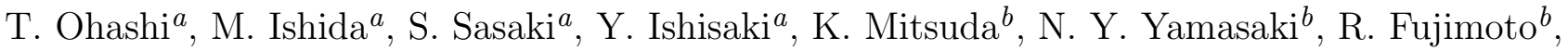 \\ Y. Takei ${ }^{b}$, Y. Tawara ${ }^{c}$, A. Furuzawa ${ }^{c}$, Y. Suto ${ }^{d}$, K. Yoshikawa ${ }^{d}$, H. Kawahara ${ }^{d}$, N. Kawai ${ }^{e}$,

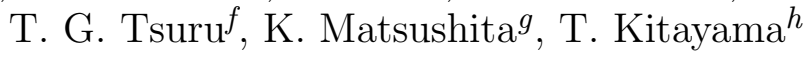 \\ ${ }^{a}$ Department of Physics, Tokyo Metropolitan University, 1-1 Minami-Ohsawa, Hachioji, \\ Tokyo 192-0397, Japan \\ ${ }^{b}$ Institute of Space and Astronautical Science, Japan Aerospace Exploration Agency, 3-1-1, \\ Yoshinodai, Sagamihara, Kanagawa 229-8510, Japan \\ ${ }^{c}$ Department of Astrophysics, Nagoya University, Furho-cho, Chikusa-ku, Nagoya 464-8602 , \\ Japan \\ ${ }^{d}$ Department of Physics, University of Tokyo, 7-3-1 Hongo, Bunkyo-ku, Tokyo 113-0033, Japan \\ e Department of Physics, Tokyo Institute of Technology, 2-12-1 Ookayama, Meguro-ku, \\ Tokyo 152-8551, Japan \\ ${ }^{f}$ Department of Physics, Kyoto University, Kitashirakawa, Sakyo-ku, Kyoto 606-8502, Japan \\ $g$ Department of Physics, Tokyo University of Science, 1-3 Kagurazaka, Shinjuku-ku, \\ Tokyo 162-8601, Japan \\ ${ }^{h}$ Department of Physics, Toho University, 2-2-1 Miyama, Funabashi, Chiba 274-8510, Japan
}

\begin{abstract}
We present our proposal for a small X-ray mission DIOS (Diffuse Intergalactic Oxygen Surveyor), consisting of a 4-stage X-ray telescope and an array of TES microcalorimeters, cooled with mechanical coolers, with a total weight of about $400 \mathrm{~kg}$. The mission will perform survey observations of warm-hot intergalactic medium using OVII and OVIII emission lines, with the energy coverage up to $1.5 \mathrm{keV}$. The wide field of view of about $50^{\prime}$ diameter, superior energy resolution close to $2 \mathrm{eV} \mathrm{FWHM,} \mathrm{and} \mathrm{very} \mathrm{low} \mathrm{background} \mathrm{will} \mathrm{together} \mathrm{enable} \mathrm{us} \mathrm{a}$ wide range of science for diffuse X-ray sources. We briefly describe the design of the satellite, performance of the subsystems and the expected results.
\end{abstract}

Keywords: intergalactic medium, X-ray spectra, oxygen lines, microcalorimeters, mechanical coolers, X-ray telescope, small mission

\section{INTRODUCTION}

The microcalorimeter experiment XRS on-board Suzaku (Astro-E2), which was launched in July 2005, has suffered from the loss of liquid helium after about a month from the launch. However, the instrument was cooled down to $60 \mathrm{mK}$ and the energy resolution of $7 \mathrm{eV}$ was obtained in the orbit for about 2 weeks. This is a significant achievement and proves that the XRS team has developed enough technologies to carry out the microcalorimeter experiment in space. There is no doubt that microcalorimeters will be the key instrument in the future X-ray astronomy.

The study of warm-hot intergalactic medium (WHIM) is the remaining frontier of X-ray astronomy. The importance of the observational study of WHIM can be summarized as follows.

1. It is certain that baryons carry $4 \%$ of the energy density in the universe, and measurements of Lyman $\alpha$ forests give consistent results. However, less than half of the baryonic matter is directly probed in the present universe. ${ }^{1}$ Numerical simulations predict that WHIM carries about $50 \%$ of baryons. Direct detection of WHIM will solve the problem of missing baryon, and the cosmic baryon budget will be fully understood.

Further author information: (Send correspondence to T. Ohashi.)

T. Ohashi: E-mail: ohashi@phys.metro-u.ac.jp, Telephone: +81-426-77-2492 
2. WHIM is the best tracer of the large-scale structure of the universe. Galaxies, by optical surveys, distribute rather sparsely in probing the continuous large-scale structure and clusters of galaxies, by X-rays, only show us the densest part of the filamentary structure. WHIM would reveal the faint part of the filament and enable us to see the structure of dark matter directly.

3. WHIM is also an important probe for the thermal history of the universe. WHIM has been ionized, heated and metal-enriched through the past star/galaxy/structure-formation processes, therefore thermal and chemical properties of the WHIM and their evolution with redshift would give us unique information in looking into the thermal history of the universe.

WHIM has been detected as absorption lines in the UV and X-ray spectra of bright AGNs. ${ }^{2}$ Recent Chandra detection of the redshifted oxygen absorption lines in the spectra of Mrk421 is the most remarkable example. ${ }^{3}$ However, absorption study does not give us full information on the spatial distribution of WHIM. It is needless to say that emission from the WHIM gives us an entirely new information, in particular about the spatial structure based on survey observations. Another point in measuring both WHIM emission and absorption in the same region is that one can solve the density $(n)$ and actual size $(L)$ of the cloud, because emission scales as $n^{2} L^{3}$ and absorption as $n L$. This may turn out to be a powerful method to look into the structure of distant gas clouds.

Since the emissivity scales as $n^{2}$, the continuum emission from the WHIM will be completely masked by the strong thermal emission from the Milky-Way hot gas which is brighter by about 2 orders of magnitude. The only possible way to detect WHIM emission is through the oxygen lines, since redshifted WHIM lines can be separated from the strong Galactic lines with energy resolution better than several eV. The existing X-ray missions are not sensitive enough, since grating spectrometers are inappropriate to cover a large sky region, for example. The observation of oxygen emission lines from the WHIM is only possible with non dispersive device like microcalorimeters. This is the reason why we propose a dedicated small mission to explore the structure of diffuse intergalactic medium, called DIOS (Diffuse Intergalactic Oxygen Surveyor), which we hope to be launched around 2010.

As described below, this mission naturally provides a very low background. We can expect very rich unique science from this mission besides the survey of WHIM.

\section{SPACECRAFT}

The view of the DIOS spacecraft is shown in Fig. 1 and main parameters of the satellite are listed in Table 1. The spacecraft will weigh about $400 \mathrm{~kg}$, out of which the payload takes $\sim 280 \mathrm{~kg}$. This mass enables the satellite to be launched as a piggy-back or sub-payload in $\mathrm{H} 2$ or Ariane rockets. It is also possible for a launch with the new ISAS rocket, such as M-V light. The size in the launch configuration is $1.5 \times 1.5 \times 1.2 \mathrm{~m}$, and one side will be expanded to about $6 \mathrm{~m}$ after the paddle deployment.

The total power is $500 \mathrm{~W}$, of which $300 \mathrm{~W}$ is consumed by the payload. The nominal orbit is a near-earth circular one with an altitude of $550 \mathrm{~km}$, in the case of launch with the ISAS rocket. An alternative choice of the orbit under consideration is an eccentric geostationary transfer orbit. This orbit gives a lower heat input from the earth and relaxes the thermal design of the satellite, and would enhance the launch opportunity to be carried as a sub-payload with geostationary satellites. One significant problem is the increased particle background as recognized with Chandra and XMM-Newton. In the soft energy range below $1 \mathrm{keV}$, electrons can be a major source of background.

The attitude will be 3 -axis stabilized with momentum wheels. Typical pointing accuracy will be about $10^{\prime \prime}$. The allowed range for the direction of the field of view is $90^{\circ} \pm 25^{\circ}$ from the sun direction. There is a radiator panel looking in the direction perpendicular to both sun and the pointing directions. To avoid the earth center covering the radiator direction, the pointing direction of the satellite will be rotated by about $180^{\circ}$ in every orbit. This attitude operation will take 6 minutes. Therefore, almost opposite sky directions will be observed at the same time. With this constraint, any position in the sky can be accessed within half a year, and the ecliptic poles are accessible throughout the year. The accessible sky regions and observable length in a year are shown in Fig. 2 Clearly a large part of the sky can be covered with DIOS, enabling not only the WHIM survey but also large-scale mapping observations of the hot Galactic interstellar medium. 


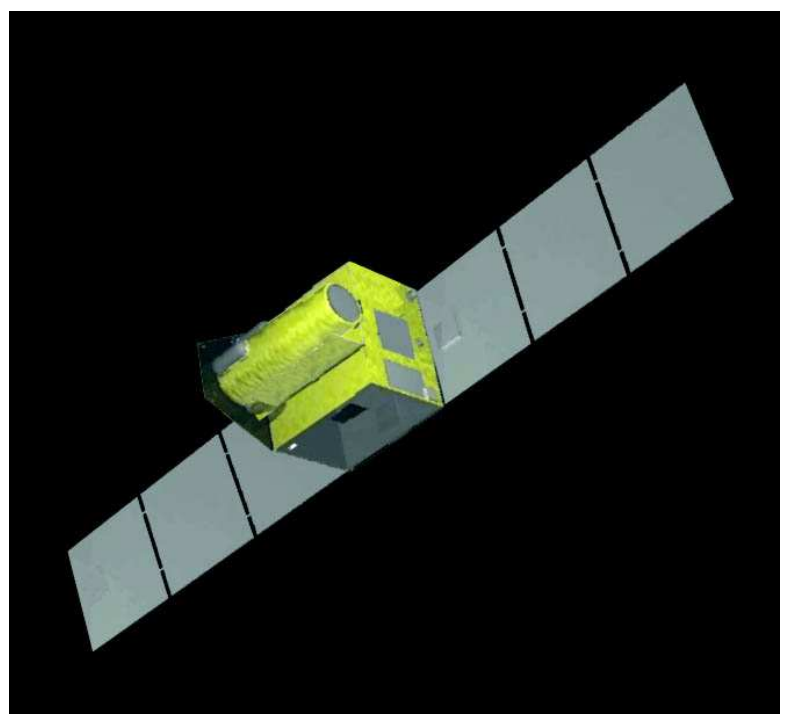

Table 1. Parameters of the observing instruments on board DIOS

\begin{tabular}{|l|l|}
\hline Effective area & $>100 \mathrm{~cm}^{2}$ \\
Field of view & $50^{\prime}$ diameter \\
$S \Omega$ & $\sim 100 \mathrm{~cm}^{2} \mathrm{deg}^{2}$ \\
Angular resolution & $3^{\prime}(16 \times 16$ pixels $)$ \\
Energy resolution & $<5 \mathrm{eV}(\mathrm{FWHM})$ \\
Energy range & $0.1-1.5 \mathrm{keV}$ \\
Observing life & $>5 \mathrm{yr}$ \\
\hline
\end{tabular}

Figure 1. The DIOS spacecraft. The length of the solar paddle is $6 \mathrm{~m}$.
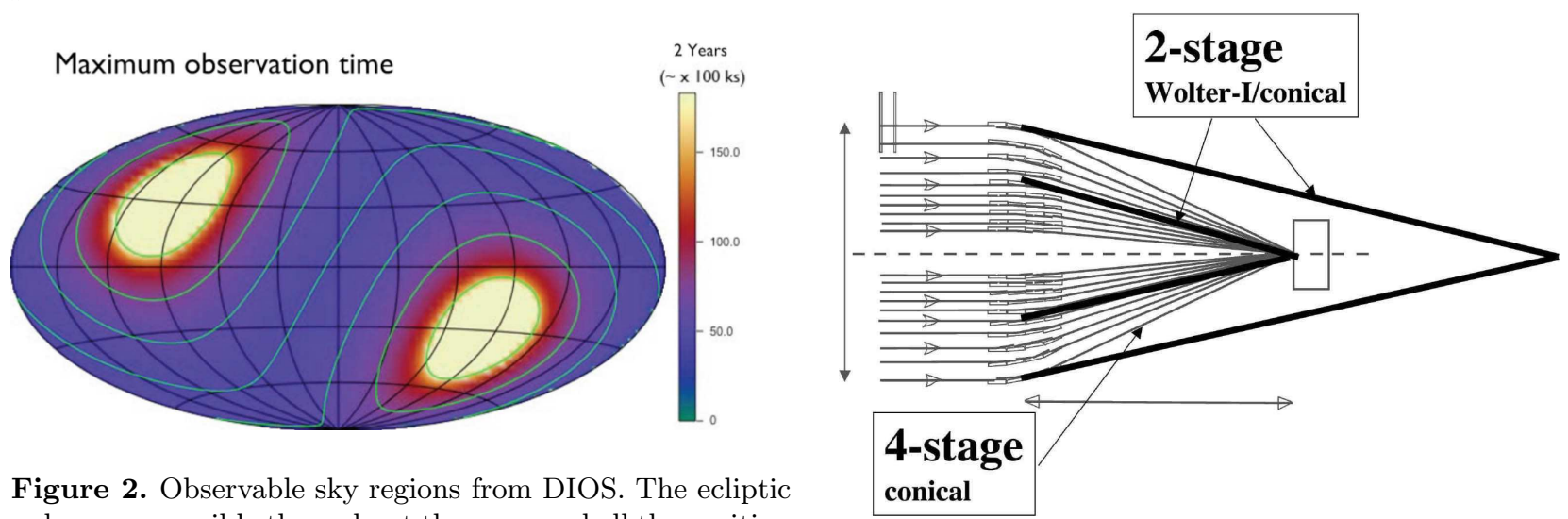

Figure 2. Observable sky regions from DIOS. The ecliptic poles are accessible throughout the year, and all the position can be observed once in a half year period.

Figure 3. Concept of the 4-stage reflection telescope.

\section{INSTRUMENTS}

Several new technologies will be introduced in the DIOS mission. The 4-stage X-ray telescope FXT (Four-stage $\mathrm{X}$-ray Telescope) is the first new feature. ${ }^{4}$ The details of the mirror development activity will be reported in the same volume. ${ }^{5}$ As shown in Fig. 3 incident X-rays are reflected 4 times by thin-foil mirrors and are focused at about $70 \mathrm{~cm}$ from the mirror level. The thin-foil technique was first used in BBXRT and then successfully used for ASCA and Suzaku. For the oxygen lines at $\sim 0.6 \mathrm{keV}$, the reflectivity of the mirror surface is close to $80 \%$ and the reduction of the effective area is not a serious problem. The 4 -stage reflection gives the focal length about half as long as the usual 2-stage design, and saves the volume and weight of the satellite substantially. Also, a small focal plane detector can cover a relatively wide field of view, which is a great advantage for the TES calorimeter array.

In our basic design, the outer diameter of the mirror and the effective area at $0.6 \mathrm{keV}$ are $50 \mathrm{~cm}$ and 400 $\mathrm{cm}^{2}$, respectively. Ray-tracing simulation indicates that the angular resolution is $2^{\prime}$ (half-power diameter), and the image quality does not show significant degradation at an offset angle $30^{\prime}$. Therefore, FXT really gives the field of view of about 1 degree. The reflectivity will be optimized by employing a multi-layer coating on the mirror surface. Al and Pt bilayer will give a good reflectivity up to $1.5 \mathrm{keV}$ (see Fig. 4), which will enable us to 
observe $\mathrm{Ne}, \mathrm{Fe}-\mathrm{L}$ and $\mathrm{Mg}$ lines. However, direct coating of $\mathrm{Al}$ and $\mathrm{Pt}$ does not work since this combination does not make a stable multilayers. Appropriate choice of the multilayer material will give an energy coverage up to $1.5 \mathrm{keV}$ (see Tawara et al. ${ }^{5}$ for details). With this technique, we can perform a spectral study of hot plasmas with a temperature up to a few $\mathrm{keV}$ from the DIOS. Most of clusters of galaxies and supernova remnants will be good targets.

Another advantage of the FXT design is the very low background. The smaller detector size for a given angular size, as a result from the short focal length, means that the internal background is very low. In fact, the focal length is about 10 times shorter than the XMM-Newton mirror, for example, indicating that 100 times smaller detector sees the same sky region with roughly 10 times smaller effective area. The low-earth orbit of DIOS also helps observation under the low background condition as well. Therefore, if we include all these factors, the background of DIOS would be more than 10 times lower than the level of Chandra and XMM-Newton in observing the same sky region. This makes DIOS a very powerful satellite to look at the WHIM and other faint emission such as outer regions of clusters of galaxies.

The focal plane instrument XSA (X-ray Spectrometer Array) is an array of TES microcalorimeters, whose development in Japan is a collaboration with Waseda University, Seiko Instruments Inc., and Mitsubishi Heavy Industries. The array consists of $16 \times 16$ pixels covering an area of about $1 \mathrm{~cm}$ square. The corresponding field of view is $50^{\prime}$. XSA will have an energy resolution better than $5 \mathrm{eV}$ FWHM at $0.6 \mathrm{keV}$. An example of the pulse-height spectra obtained for $5.9 \mathrm{keV}$ X-rays with our TES calorimeter, which is a single pixel type made with Ti-Au bilayer with a Au X-ray absorber, is shown in Fig. 囵 ${ }^{6}$ The measured resolution is $6.3 \mathrm{eV}$ FWHM. Since the energy range of XSA is below $1.5 \mathrm{keV}$, the required heat capacity of the X-ray absorber is significantly low. Therefore, a significantly better energy resolution will be achievable in the soft X-ray range.

We are now developing several new techniques toward the multi-pixel operation of TES calorimeters. ${ }^{7,8}$ X-ray absorbing material under consideration is $\mathrm{Bi}$, which has low heat capacity and does not produce long-life quasi particles in the absorber. We are testing an electro-plating method to build a $16 \times 16$ absorber array with a pixel size $\sim 0.5 \times 0.5 \mathrm{~mm}$ supported by a thin stem (see Fig. [6 for our test model made with Sn). In this figure, all the pixels have wirings equipped and they can all operate as microcalorimeters. Several pixels indeed show $\mathrm{X}$-ray sensitivity, however absorbers are not mechanically stable under the thermal cycle. We are looking into other stress-free method to mount the absorber structure.

For the signal readout, efficient multiplexing of the signals is essential to take all the data out from the cold stage. We are trying to add the signals, e.g. 8 of them, in frequency space by driving the TES calorimeters with AC bias at different frequencies for different pixels. ${ }^{9,10}$ The summed signals are retrieved by single wire and then demodulated by a room-temperature electronics. The SQUID needs to be operated at $1 \mathrm{MHz}$ to obtain a dynamic range in the frequency space. We have produced 8-input SQUID (see Fig. (7), in collaboration with the Seiko Instruments Inc., to add the TES signals, and it is under a performance test now. An efficient thermal shield with high soft X-ray transmission is also an essential part, and a layer of 5 parylene films with $0.1 \mu \mathrm{m}$ thickness each has been successfully used in the laboratory.

Another important feature of DIOS is the cryogen-free cooling system. As shown in Fig. [ 8 we are considering a serial connection of different types of coolers to achieve the $\sim 50 \mathrm{mK}$ operation for XSA within the available power budget. The outside panel is equipped with a radiator which has $1.5 \mathrm{~m}^{2}$ area. The first stage of the cooler is a 2-stage Stirling cooler which takes the temperature down to $20 \mathrm{~K}$, and then ${ }^{3} \mathrm{He}$ Joule-Thomson cooler reduces it to $1.8 \mathrm{~K}$ as the second stage. Then a 2-stage adiabatic demagnetization refrigerator obtains the operating temperature at $50 \mathrm{mK}$. Since no cryogen is involved, this cooling system ensures an unlimited observing life in the orbit, which is a significant advantage of DIOS. The XSA system is subject to a warm launch, therefore we have to allow for the initial cooling of the system for the first 1-2 months in the orbit. We note that Stirling coolers are operating successfully in Suzaku continuously for more than 10 months in space. The coolers are under development with Sumitomo Heavy Industry.

\section{OBSERVATION}

The main purpose of DIOS is a sky survey of WHIM using oxygen K emission lines, based on the superior energy resolution and a large field of view. Yoshikawa et al. ${ }^{11,12}$ carried out detailed simulation for the spectra expected 


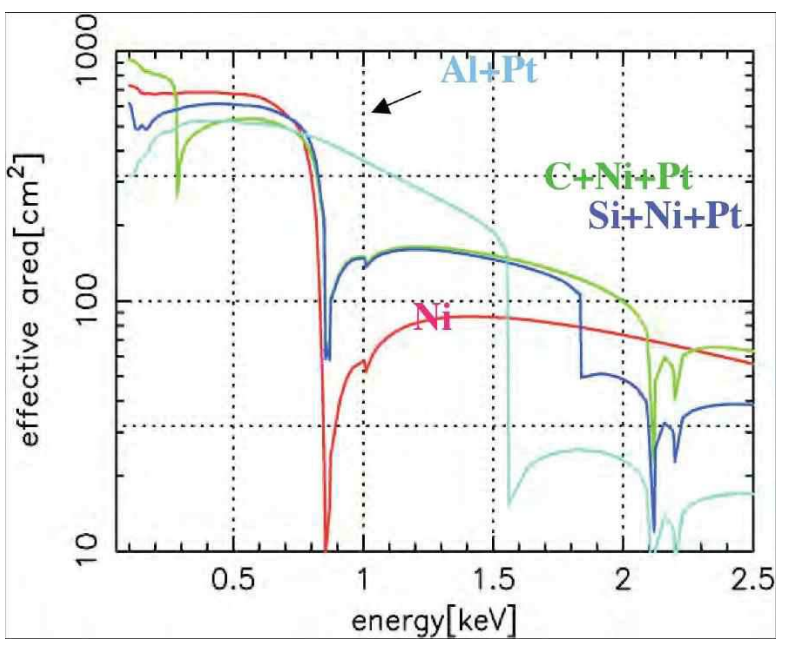

Figure 4. Effective area as a function of energy for various multi-layer coatings. $\mathrm{Al}$ and $\mathrm{Pt}$ bilayer gives a good performance up to $1.5 \mathrm{keV}$. See Tawara et al. in this volume for details.

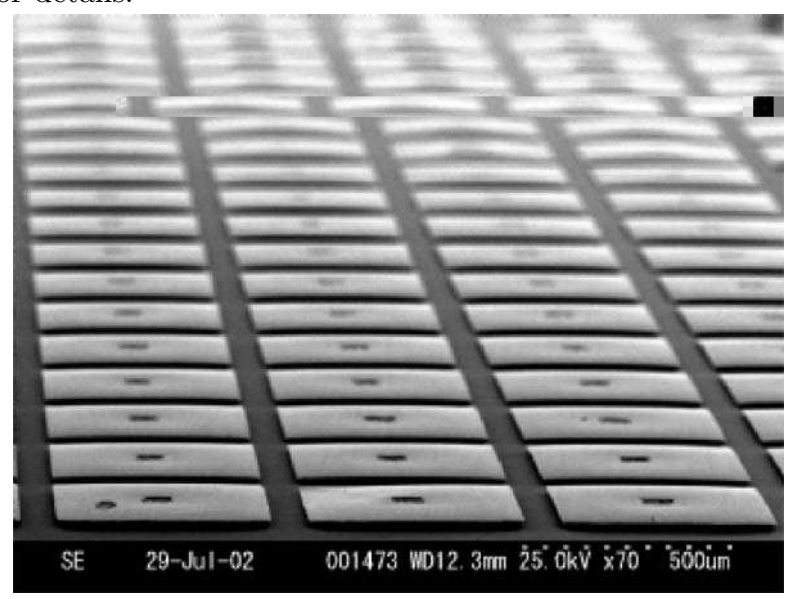

Figure 6. Mechanical model of the 256 pixel absorber for the TES array, produced by a Sn plating. Size of one pixel is $500 \mu \mathrm{m}$.

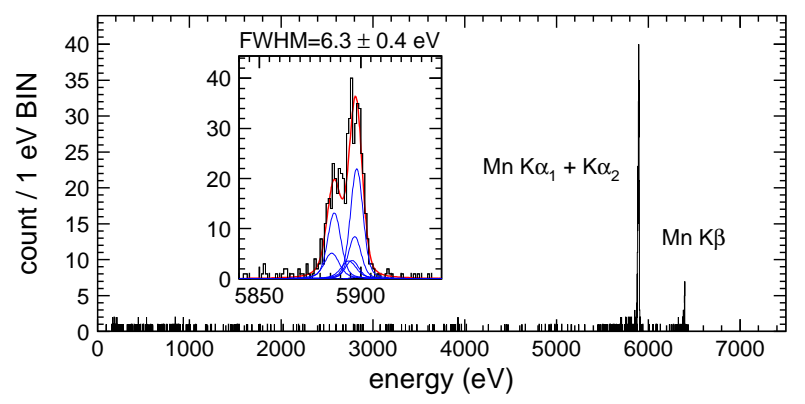

Figure 5. Pulse-height spectrum for $\mathrm{Mn}-\mathrm{K} \alpha$ X-rays obtained by a single pixel Ti-Au TES microcalorimeter.

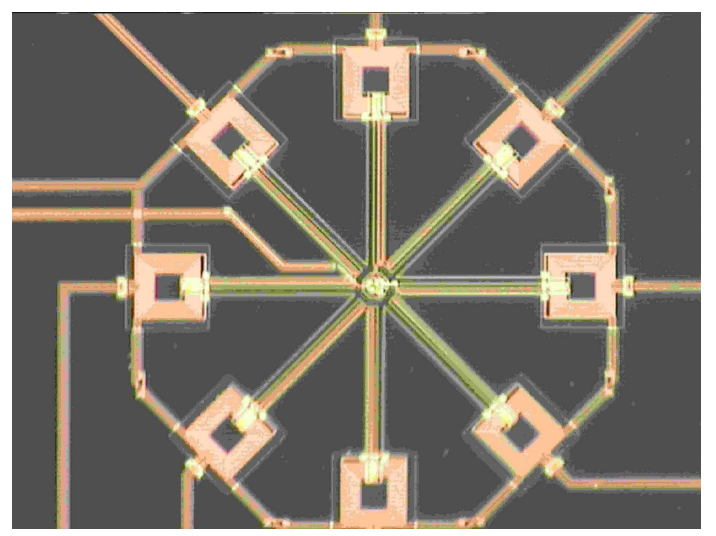

Figure 7. Test model of the 8-input SQUID.

from the DIOS observations. It is based on a cosmological hydrodynamic simulation for a cube of $75 h^{-1} \mathrm{Mpc}$ size, which corresponds to an angular size of $5^{\circ}$ at a redshift 0.3 . The cosmological evolution of intergalactic medium is included. The metallicity of the gas is explicitly given, and the effective oxygen abundance is $0.1-0.2$ solar. The WHIM emission in a redshift range $z=0-0.3$ is accumulated along various trial lines of sight in the sky and the resultant X-ray spectra are calculated. In this simulation, WHIM is assumed to be under a collisional ionization equilibrium.

The simulation shows that each pointing will need typically 100 ksec to obtain enough oxygen photons from the IGM. Fig. 9] shows the expected pulse-height spectra for different sky regions. The strong emission lines from our Galaxy are included in the simulated observation and then subtracted in this plot. So, the statistical fluctuation of the data includes this effect. Strong oxygen lines are expected from relatively dense WHIM regions, which are either close to clusters of galaxies or containing groups of galaxies (such as the region B). Note that the hot interstellar medium in our galaxy produces typically 100 times stronger line emission, which can be separated from the redshifted WHIM lines with the energy resolution of the XSA. When the data quality is 


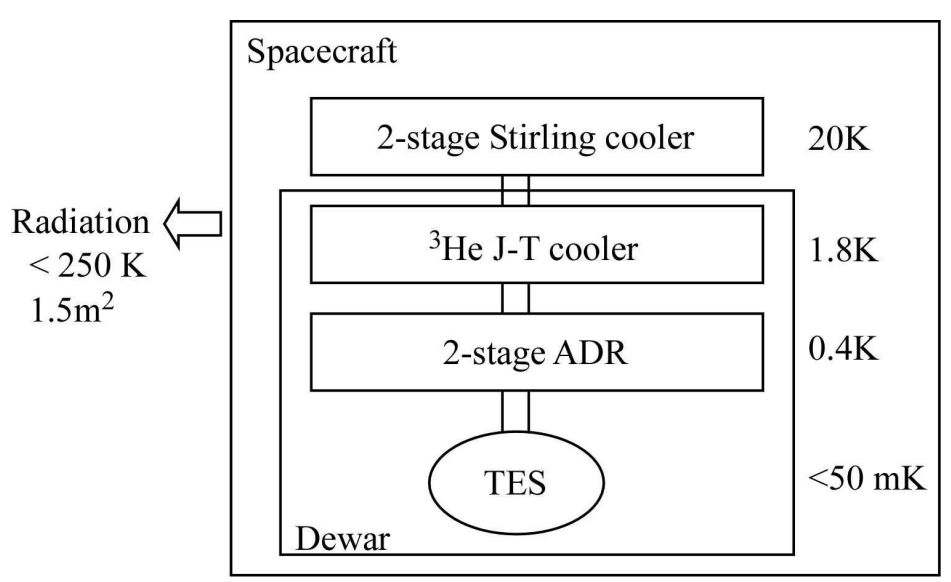

Figure 8. Cooling system for the TES calorimeter system.

high, the lines in the OVII triplet are clearly resolved and we will be able to measure the temperature directly. These 3 lines can also be used to separate individual plasma components when several emission regions in the same line of sight but different redshifts are observed together.

The fraction of baryons probed by the detection limit of DIOS is shown in Fig. 10] in which mass fraction of baryons are plotted as a function of detection flux. For a nominal detection limit of DIOS, i.e. $10^{-10}-10^{-11}$ erg $\mathrm{cm}^{-2} \mathrm{~s}^{-1} \mathrm{sr}^{-1}$, about $20 \%$ of all the baryons will be detected. We note that OVIII line is more efficient than OVII line, since the temperature range in which the line is emitted is wider. Aside from the hot cluster gas $\left(T>10^{7} \mathrm{~K}\right)$, DIOS can detect roughly half of the missing baryons, namely the gas in the temperature range $10^{6}-10^{7} \mathrm{~K}$. This indicates that, even the DIOS satellite itself is quite small, the mission can carry out the expected science.

The DIOS mission can also perform a mapping observation of the hot interstellar medium in our galaxy. The high energy resolution $(\Delta E<5 \mathrm{eV})$ will reveal the Doppler shifts of the hot interstellar gas with a velocity $\sim 100$ $\mathrm{km} \mathrm{s}^{-1}$. The observations from DIOS can confirm the large-scale outflow and falling-in of the hot bubbles in our galaxy (galactic fountain). Apart from this science, the energy coverage up to $1.5 \mathrm{keV}$ with superior resolution, combined with the wide field of view ( $50^{\prime}$ diameter) and the extremely low internal background, make DIOS a very powerful satellite for the study of diffuse hot gas in various scales. Hot-gas properties near the virial radius of clusters of galaxies remain highly uncertain even with the Chandra and XMM-Newton observations. DIOS will give us a clear answer about the temperature and metallicity in this region and will give new constraints on the shock heating of the infalling gas. Also, study of dynamical processes (shocks and bullets) in supernova remnants will advance significantly with the high resolution spectroscopy from DIOS. In this view, the capability of DIOS is highly complementary to the existing and planned large X-ray observatories.

Effective observing time will be approximately $40 \mathrm{ksec}$ per day for a near earth orbit. So if we spend 100 ksec in each pointing position, a sky map for an area of $10^{\circ} \times 10^{\circ}$ can be produced in about a year. This angular size is enough to see the large-scale structure of the universe at $z<0.3$. This survey observation of a limited sky region will be the first task of DIOS. Since oxygen lines from the galactic ISM are stronger by roughly 2 orders of magnitude, $1 \mathrm{ksec}$ in each point is enough to make a large map of the ISM distribution. We plan to devote the second year for a survey of a $100^{\circ} \times 100^{\circ}$ sky for the galactic ISM. After these 2 years, further deeper observations of the IGM as well as of various selected objects can be performed. Fig. 111 compares $S \Omega$, the grasp as the product of effective area and solid angle, and energy resolution among different instruments in the X-ray missions which are planned or already operating. Clearly, DIOS will achieve the highest sensitivity for soft X-ray lines from extended objects, except for the larger mission, NEW, based on the similar concept. 


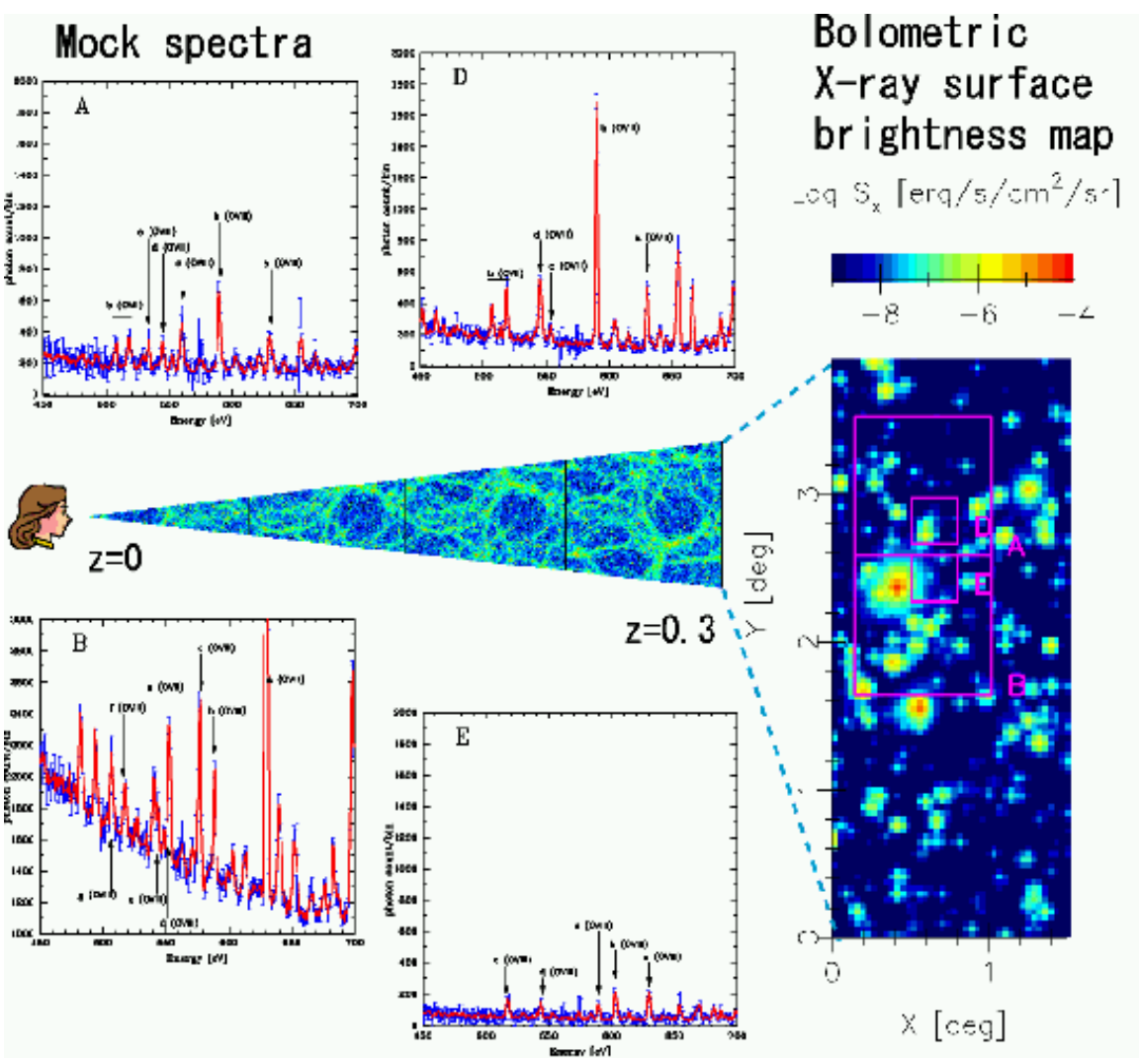

Figure 9. Simulated observations of WHIM observed from DIOS. ${ }^{11}$ Typical observation time is $100 \mathrm{ksec}$. Foreground Galactic emission is included in the simulation and subtracted from the data.

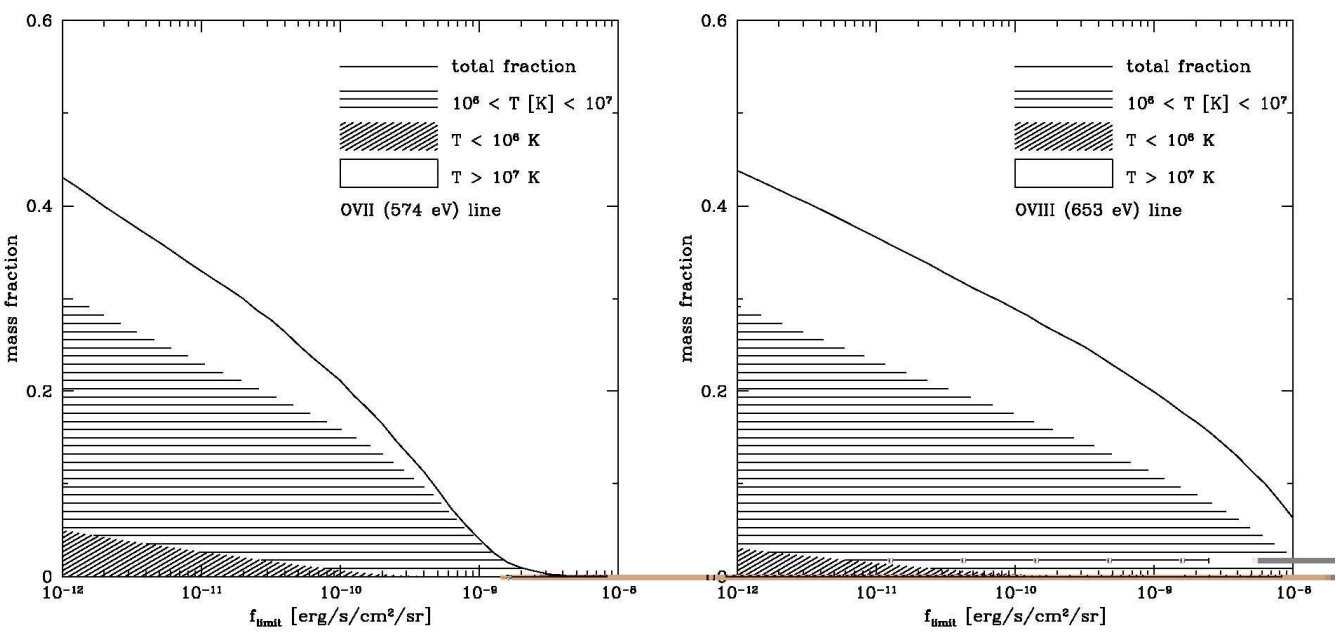

Figure 10. Probed fraction of baryons plotted as a function of detection limit. ${ }^{12}$ The left panel is for OVII line and the right one for OVIII line, respectively.

\section{PROSPECT}

DIOS has been proposed to ISAS/JAXA in 2005 together with several tens of other small missions. This year, 5 proposals were chosen as a pilot example of small missions to be reported to JAXA headquarter. DIOS is one of them, and only one for the astronomy mission. Even though the program of small missions has not started in JAXA, DIOS may have a chance of fairly early opportunity. On the other hand, Japanese X-ray 


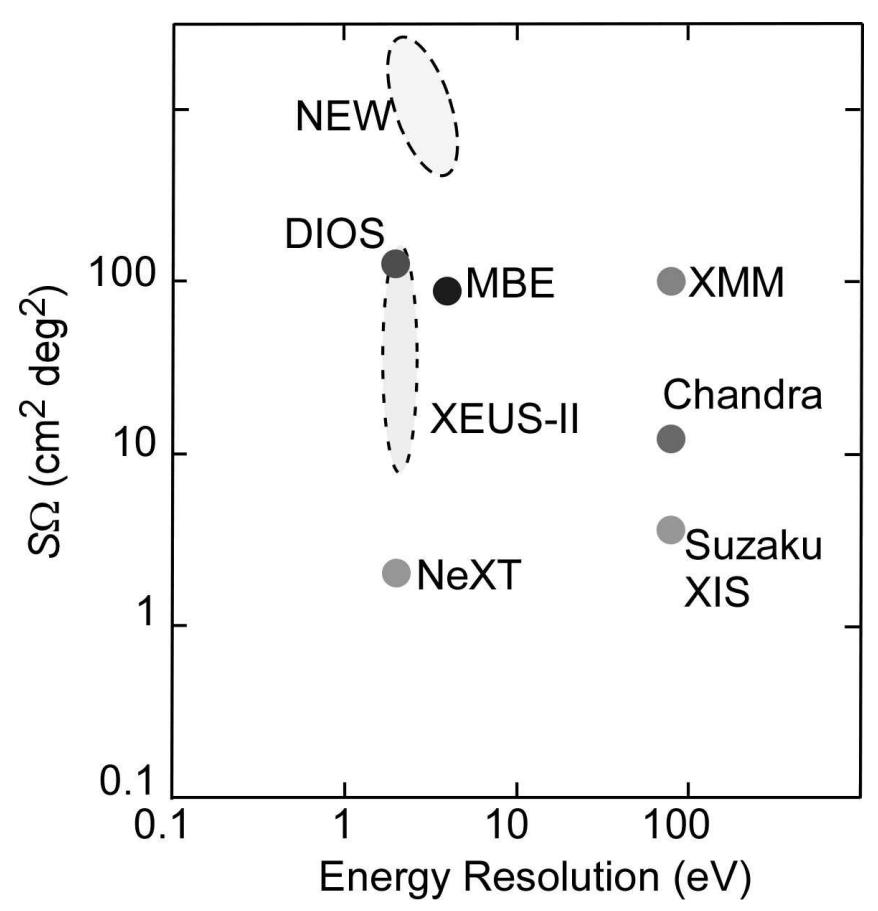

Figure 11. Comparison of $S \Omega$ and energy resolution for spectroscopic instruments (CCDs and microcalorimeters) for planned and operating X-ray satellites.

group is proposing NeXT, which is a larger mission also carrying microcalorimeters. In the case that the two mission might cause some kind conflict, we would think of down sizing of the DIOS mission. At the same time, we are exploring international collaboration. Dutch mission NEW and Italian ESTREMO share the same scientific purpose with DIOS. It is quite likely that hardware team would collaborate and concentrate on the most promising single mission.

\section{REFERENCES}

1. M. Fukugita, C. J. Hogan, and P. J. E. Peebles, "The cosmic baryon budget," ApJ 503, p. 518, 1998.

2. T. M. Tripp, D. V. Bowen, K. R. Sembach, E. B. Jenkins, B. D. Savage, and P. Richter, "Missing baryons in the warm-hot intergalactic medium," astro-ph/0411151, 2004.

3. F. Nicastro, S. Mathur, M. Elvis, J. Drake, F. Fiore, T. Fang, A. Fruscione, Y. Krongold, H. Marshall, and R. Williams, "Chandra detection of the first x-ray forest along the line of sight to Markarian 421," ApJ 629, p. 700, 2005.

4. Y. Tawara, Y. Ogasaka, K. Tamura, and A. Furuzawa, "Development of four-stage x-ray telescope for wide-field fine spectroscopic mission," SPIE 5168, p. 386, 2004.

5. Y. Tawara, A. Furuzawa, Y. Ogasaka, R. Shibata, and K. Tamura, "The demonstration model of four-stage X-ray telescope for DIOS," SPIE 6266, 2006.

6. Y. Ishisaki, U. Morita, T. Koga, K. Sato, T. Ohashi, K. Mitsuda, N. Y. Yamasaki, R. Fujimoto, N. Iyomoto, T. Oshima, K. Futamoto, Y. Takei, T. Ichitsubo, T. Fujimori, S. Shoji, H. Kudo, T. Nakamura, T. Arakawa, T. Osaka, T. Homma, H. Sato, H. Kobayashi, K. Mori, K. Tanaka, T. Morooka, S. Nakayama, K. Chinone, Y. Kuroda, M. Onishi, and K. Otake, "Present performance of a single pixel Ti/Au bilayer TES calorimeter," SPIE 4851, p. 831, 2003.

7. Y. Ishisaki, T. Ohashi, T. Oshima, U. Morita, K. Shinozaki, K. Sato, K. Mitsuda, N. Y. Yamasaki, R. Fujimoto, Y. Takei, H. Sato, N. Takahashi, T. Homma, and T. Osaka, "Development of a microcalorimeter array for the diffuse intergalactic oxygen surveyor (DIOS) mission," SPIE 5501, p. 123, 2004. 
8. U. Morita, Y. Yamakawa, T. Fujimori, Y. Ishisaki, T. Ohashi, Y. Takei, K. Yoshida, T. Yoshino, K. Mitsuda, N. Y. Yamasaki, R. Fujimoto, H. Sato, Y. Minoura, N. Takahashi, T. Homma, S. Shoji, Y. Kuroda, and M. Onishi, "Evaluation of 256-pixel tes microcalorimeter arrays with electrodeposited Bi absorbers," Nucl. Instr. and Meth. A 559, p. 539, 2006.

9. N. Iyomoto, T. Ichitsubo, K. Mitsuda, N. Y. Yamasaki, R. Fujimoto, T. Oshima, K. Futamoto, Y. Takei, T. Fujimori, K. Yoshida, Y. Ishisaki, U. Morita, T. Koga, K. Shinozaki, K. Sato, N. Takai, T. Ohashi, T. Miyazaki, S. Nakayama, K. Tanaka, T. Morooka, and K. Chinone, "Frequency-domain multiplexing of tes microcalorimeter array with CABBAGE," Nucl. Instr. and Meth. A 520, p. 566, 2004.

10. N. Y. Yamasaki, K. Masui, K. Mitsuda, T. Morooka, S. Nakayama, and Y. Takei, "Design of frequency domain multiplexing of TES signals by multi-input SQUIDs," Nucl. Instr. and Meth. A 559, p. 790, 2006.

11. K. Yoshikawa, N. Y. Yamasaki, Y. Suto, T. Ohashi, K. Mitsuda, Y. Tawara, and A. Furuzawa, "Detectability of the warm/hot intergalactic medium through emission lines of O VII and O VIII," PASJ 55, p. 879, 2003.

12. K. Yoshikawa, K. Dolag, Y. Suto, S. Sasaki, N. Y. Yamasaki, T. Ohashi, K. Mitsuda, Y. Tawara, R. Fujimoto, T. Furusho, A. Furuzawa, M. Ishida, Y. Ishisaki, and Y. Takei, "Locating the warm-hot intergalactic medium in the simulated local universe," PASJ 56, p. 939, 2004. 